\title{
Study the Effect of Organic and Inorganic Sources of Nitrogen on Growth and Yield of Kharif Pearl Millet
}

\author{
Shobha Kumari*, Vikram Singh, Neha and Preeti Choudhary \\ Department of Agronomy, Sam Higginbottom Institute of Agriculture, Technology and Sciences, \\ (Formerly Allahabad Agricultural Institute), (Deemed to-be-University), \\ Allahabad - U.P. - 211 007, India \\ *Corresponding author
}

\section{A B S T R A C T}

\begin{tabular}{|l|}
\hline K e y w o r d s \\
Kharif hybrid \\
pearlmillet, \\
Levels of \\
nitrogen, Plant \\
height, Weight \\
and yield.
\end{tabular}

Among the response of different hybrids to levels of nitrogen, treatment $\mathrm{T}_{9}$ i.e., $\mathrm{V}_{3}+$ 100:45:45 kg ha ${ }^{-1} \mathrm{NPK}$, recorded maximum plant height $(175.30 \mathrm{~cm})$, number of tillers plant $^{-1}(3.67)$, dry weight $(47.73 \mathrm{~g})$, crop growth rate $\left(1.53 \mathrm{~g} \mathrm{~m}^{-2}\right.$ day $\left.^{-1}\right)$, relative growth rate $\left(0.052 \mathrm{~g} \mathrm{~g}^{-1}\right.$ day $\left.^{-1}\right)$, number of ear plant ${ }^{-1} 2.37$, length of ear $(27.52 \mathrm{~cm})$, number of Grains $\operatorname{ear}^{-1}(2406.49)$ 'test weight $(10.29 \mathrm{~g})$, grain yield $\left(3.72 \mathrm{t} \mathrm{ha}^{-1}\right)$, straw yield(6.98 $\left.\mathrm{t} \mathrm{ha}{ }^{-1}\right)$, protein content $(13.43 \%)$ ) and harvest index $(36.15 \%)$. Whereas the lowest value in terms of plant height $(164.47 \mathrm{~cm})$, number of tillers $\left(2.60\right.$ plant $\left.^{-1}\right)$, dry weight $(40.80 \mathrm{~g})$, crop growth rate $\left(1.30 \mathrm{~g} \mathrm{~m}^{-2} \mathrm{day}^{-1}\right)$, relative growth rate $\left(0.045 \mathrm{~g} \mathrm{~g}^{-1}\right.$ day $\left.^{-1}\right)$, number of ear $(1.37$ plant $\left.{ }^{-1}\right)$, grain yield $\left(2.47 \mathrm{tha}^{-1}\right)$ and straw yield $\left(4.62 \mathrm{t} \mathrm{ha}^{-1}\right)$ was observed in the treatment $\mathrm{T}_{1}$ i.e., $\left.\mathrm{V}_{1}+80: 45: 45 \mathrm{~kg} \mathrm{ha}^{-1} \mathrm{NPK}\right)$. Further, ear length of $(21.16 \mathrm{~cm})$ was observed in the treatment $\mathrm{T}_{8}$ i.e., $\left.\mathrm{V}_{3}+90: 45: 45 \mathrm{~kg} \mathrm{ha}^{-1} \mathrm{NPK}\right)$. In terms of number of grains (2406.49 $\left.\mathrm{ear}^{-1}\right)$, test weight $(8.04 \mathrm{~g})$ was recorded in the treatment $\mathrm{T}_{5}$ i.e., $\left.\mathrm{V}_{2}+90: 45: 45 \mathrm{~kg} \mathrm{ha}^{-1} \mathrm{NPK}\right)$. Further, protein content $(9.48 \%)$ was observed in the treatment $\mathrm{T}_{5}$ i.e., $\mathrm{V}_{2}+90: 45: 45 \mathrm{~kg}$ $\left.\mathrm{ha}^{-1} \mathrm{NPK}\right)$ and harvest index $(19.01 \%)$ respectively in the treatment $\mathrm{T}_{4}$ i.e., $\mathrm{V}_{2}+80: 45: 45$ $\left.\mathrm{kg} \mathrm{ha}^{-1} \mathrm{NPK}\right)$.

\section{Introduction}

Pearlmillet (Pennisetum glaucum L.) is largely grown for grain and fodder purpose under those situations where other crops generally fail. Pearlmillet as a food crop is limited to the developing countries in Asia, and particularly in Africa and ranked sixth in the world followed by rice, wheat, maize, barley and sorghum (Anonymous, 2010-11). The important pearlmillet growing countries are India, China, Nigeria, Pakistan, Sudan, Egypt, Arabia, and Russia. It is estimated that over $95 \%$ of pearlmillet production is used as food, the reminder being divided between animal and poultry feed (7\%), other uses (seed, bakery products, snacks, etc.) and waste. Pearlmillet is used in flat breads (roti) or mixed up to $25 \%$ with wheat flour for use in yeast breads. In India, pearlmillet is popularly known as Bajra, and it is the fourth most important cereal crop after rice, wheat and sorghum. It has the greatest potential among all the millets. The major producing states are Rajasthan (46\%), Maharashtra (19\%), Gujarat (11\%), Uttar Pradesh $(8 \%)$ and Haryana (6\%) (Sonawane et al., 2010). Nutrient management system refers to 
combined application of all nutrient source viz. Vermicompost, use of Biofertilizer (Azotobacter) and inorganic fertilizer (urea). The combined effect of organic and inorganic source of nutrient help in maintaining yield stability through correction of nutrients deficiencies, enhancing their efficiency and providing favorable soil physiological condition (Behera et al., 2007). Biofertilizers play an important role in increasing the availability of native and applied nutrients and productivity in sustainable manner. Azotobacter is a free-living nitrogen fixing bacteria. It has been reported to fix about 20 $\mathrm{kg} \mathrm{N} \mathrm{ha}{ }^{-1}$ per year in a field of non-legume crop and also secretes some growth promoting substances (Kumawat and Jat, 2007).

\section{Materials and Methods}

The experiment entitled, "Study the effect of organic and inorganic sources of nitrogen on growth and yield of kharif pearmillet" was carried out at Crop Research Farm, Department of Agronomy, Sam Higginbottom Institute of Agriculture, Technology and Sciences, (Deemed University), (Formally Allahabad Agricultural Institute), Allahabad.

During the kharif season of 2015, laid out in Randomized Block Design, having ten treatments and three replications. The experiment was conducted to, "Study the effect of organic and inorganic sources $f$ nitrogeon on growth and yield of kharif pearmillet". The experimental findings based on parameters are summarized below.

\section{Experimental details}

The experiment was laid out in Randomized Block Design, comprising of ten treatment combinations each replicated thrice. Treatments were randomly arranged in each replication, divided into ten plots. The treatment tested, specifications of the layout, etc are given below.

\section{Treatment combinations}

$\mathrm{T}_{1}: 100 \% \mathrm{RDN}$ through Urea

$\mathrm{T}_{2}: 100 \% \mathrm{RDN}$ through Urea + Azotobacter

$\mathrm{T}_{3}: 100 \% \mathrm{RDN}$ through VC

$\mathrm{T}_{4}: 100 \% \mathrm{RDN}$ through $\mathrm{VC}+$ Azotobacter

$\mathrm{T}_{5}: 75 \% \mathrm{RDN}$ through $\mathrm{VC}+25 \% \mathrm{~N}$ through Urea

$\mathrm{T}_{6}: 75 \% \mathrm{RDN}$ through $\mathrm{VC}+25 \% \mathrm{~N}$ through Urea + Azotobacter

$\mathrm{T}_{7}: 50 \% \mathrm{RDN}$ through $\mathrm{VC}+50 \% \mathrm{~N}$ through Urea

$\mathrm{T}_{8}: 50 \% \mathrm{RDN}$ through $\mathrm{VC}+50 \% \mathrm{~N}$ through Urea+ Azotobacter

$\mathrm{T}_{9}: 25 \% \mathrm{RDN}$ through $\mathrm{VC}+75 \% \mathrm{~N}$ through Urea

$\mathrm{T}_{10}: 25 \% \mathrm{RDN}$ through $\mathrm{VC}+75 \% \mathrm{~N}$ through Urea + Azotobacter

Recommended dose of fertilizer 120: 60: 40 $\mathrm{N}: \mathrm{P}: \mathrm{K}$

Nitrogen application in two splits ( $1 / 2$ basal and $1 / 2$ top dressing at 30 DAS).

Full dose of P\&K used as basal.

\section{Observation recorded}

\section{Pre-harvest observation}

\section{Plant height (cm)}

Height of ten plants tagged in each plot was measured from ground level to the base of the flag leaf at 20, 40, 60 and 80 DAS. 
Average value for each plant was computed and expressed in $\mathrm{cm}$.

\section{Dry weight plant $^{-1}(\mathrm{~g})$}

To study the dry matter accumulation of crops, five plants were uprooted from sampling rows from each plot randomly at 20, 40, 60 and 80 DAS. After removing the root portion, the above ground parts of each plant were first sundried, and then oven dried at $70^{\circ} \mathrm{C}$ for 72 hours. After complete drying, plants were weighed and the unit expressed in g.

\section{Crop growth rate $\left(\mathrm{g} \mathrm{m}^{-2} \mathrm{day}^{-1}\right)$}

It represents dry weight gained by a unit area of crop in a unit time expressed as $\mathrm{g} \mathrm{m}^{-2}$ day $^{-1}$ (Brown and Mayer, 1988). The values of plant dry weight at 20, 40, 60 and 80 DAS intervals were used for calculating the CGR. It was calculated with the help of following formula:

Crop Growth Rate $=\frac{\mathrm{W}_{2}-\mathrm{W}_{1}}{\mathrm{t}_{2}-\mathrm{t}_{1}}\left(\mathrm{~g} \mathrm{~m}^{-2} \mathrm{day}^{-1}\right)$

Where,

$\mathrm{W}_{1}=$ dry matter production per unit area at time $t_{1}$

$\mathrm{W}_{2}=$ dry matter production per unit area at time $t_{2}$

$\mathrm{t}_{1}=$ days to first sampling

$\mathrm{t}_{2}=$ days to second sampling

\section{Relative growth rate $\left(\mathrm{gg}^{-1} \mathrm{day}^{-1}\right)$}

It was indicates the increase in dry weight per unit dry matter over any specific time interval and it was calculated by the following equation:
Relative Growth

$$
\log _{\mathrm{e}} \mathrm{W}_{2}-\log _{\mathrm{e}} \mathrm{W}_{1}
$$

Rate $(\mathrm{RGR})=$------------------ $\left(\mathrm{g} \mathrm{g}^{-1} \mathrm{day}^{-1}\right)$

Where,

$\mathrm{W}_{1}=$ Initial dry weight of plant $(\mathrm{g})$

$\mathrm{W}_{2}=$ Final dry weight of plant $(\mathrm{g})$

$\mathrm{t}_{1}=$ Initial time period

$\mathrm{t}_{2}=$ Final time period

It is also called efficiency index (y) and can be expressed in $\mathrm{g} \mathrm{g}^{-1}$ day $^{-1}$

This parameter was calculated at different time intervals i.e., 20, 40, 60 and 80 DAS. Using the data obtained from dry weight of plants.

\section{Post-harvest observation}

\section{Length of ear (cm)}

Three ear lengths were measured by scale from each plots and the average value for each ear was computed and expressed in $\mathrm{cm}$.

\section{Test weight $(\mathrm{g})$}

A small seed sample was taken from the produce of each plots harvested and weight of 1000 -seeds from each plot was taken and the average test weight of each plot was recorded.

\section{Pearlmillet grain yield $\left(\mathrm{t} \mathrm{ha}^{-1}\right)$}

The grain yield of each plot was recorded after cleaning the threshed produce and it was converted as ( $\left.\mathrm{t} \mathrm{ha}^{-1}\right)$.

\section{Stover yield (t ha-1)}

The Stover yield was obtained by subtracting the seed yield from biological yield plot $^{-1}$ recorded earlier and then converted in terms of $\left(\mathrm{tha}^{-1}\right)$. 


\section{Harvest index (\%)}

The harvest index was worked out using feed and stover yield with the help of formula given by Donald (1962).

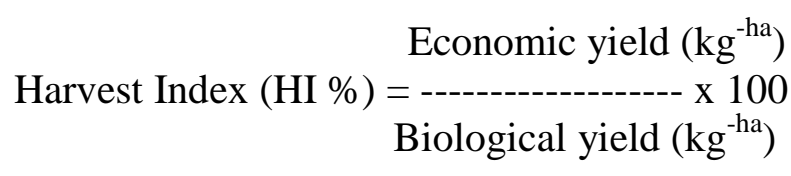

\section{Results and Discussion}

The findings of the present are presented and discussed in the following pages under appropriate headings.

Data on pre-harvest and post-harvest observations were analyzed and discussion on experimental findings has been stated.

The parameters are also illustrated with the help of graphs wherever felt essential to clarify the results.

\section{Observation recorded}

Pre-harvest observations of pearlmillet (at 20, 40, 60 and 80 DAS)

Plant height $(\mathrm{cm})$

Plant dry weight $(\mathrm{g})$

No. Tillers

Crop growth rate $\left(\mathrm{g} \mathrm{m}^{-2} \mathrm{day}^{-1}\right)$

Relative growth rate $\left(\mathrm{g} \mathrm{g}^{-1} \mathrm{day}^{-1}\right)$

\section{Post-harvest observations of pearlmillet}

Number of ear pliant ${ }^{-1}$

Length of ear $(\mathrm{cm})$

Test weight $(\mathrm{g})$

Grain yield $\left(\mathrm{t} \mathrm{ha}^{-1}\right)$

Stover yield $\left(\mathrm{t} \mathrm{ha}^{-1}\right)$

Biological yield $\left(\mathrm{t} \mathrm{ha}^{-1}\right)$

Harvest index (\%)

Protein content (\%)

\section{Growth parameters of pearlmillet}

\section{Pre-harvest observations of pearlmillet Plant height (cm)}

The observations of plant height are being presented in table 1. A perusal of that there was a steady increase in the plant height from 20 to 80 DAS. At 40,60 and 80 DAS were significant influence in plant height due to different treatments, while at 20 DAS nonsignificant. At 20 DAS, there was no significantly difference between different treatments. Maximum plant height $(86.88$ $\mathrm{cm})$ was obtained by the application of $\mathrm{T}_{1}$ (100\% RDN through Urea), whereas the lowest value $76.30 \mathrm{~cm}$ in T3 (100\% RDN through VC). At 40 DAS, there was significantly difference between different treatments. Maximum plant height (189.34 $\mathrm{cm}$ ) was obtained by the application of $\mathrm{T}_{10}$ (25\% RDN through $\mathrm{VC}+75 \% \mathrm{~N}$ through Urea + Azotobacter), whereas the lowest value $159.67 \mathrm{~cm}$ in $\mathrm{T}_{3}(100 \% \mathrm{RDN}$ through VC). However, $\mathrm{T}_{9}(25 \% \mathrm{RDN} \mathrm{VC}+75 \% \mathrm{~N}$ through Urea), $\mathrm{T}_{2}(100 \% \mathrm{RDN}$ through Urea + Azotobacter) and $\mathrm{T}_{8}(100 \%$ RDN through $\mathrm{VC}+50 \% \mathrm{~N}$ through Urea + Azotobacter) were found statistically at par with $\mathrm{T}_{10}(25 \%$ through $\mathrm{VC}+75 \% \mathrm{~N}$ through Urea + Azotobacter). At 60 DAS, there was significant difference between different treatments. Maximum plant height (243.33 $\mathrm{cm})$ was obtained by the application of $\mathrm{T}_{10}$ (25\% through $\mathrm{VC}+75 \% \mathrm{~N}$ through Urea + Azotobacter). Whereas the lowest value $226.64 \mathrm{~cm}$ in $\mathrm{T}_{3}(100 \% \mathrm{RDN}$ through $\mathrm{VC})$. However, $\mathrm{T}_{9}(25 \% \mathrm{RDN} \mathrm{VC}+75 \% \mathrm{~N}$ through Urea), $\mathrm{T}_{8}(100 \% \mathrm{RDN}$ through $\mathrm{VC}+$ $50 \% \mathrm{~N}$ through Urea + Azotobacter), and $\mathrm{T}_{1}$ (100 \% RDN through Urea) were found statistically at par with $\mathrm{T}_{10}(25 \%$ through VC $+75 \% \mathrm{~N}$ through Urea + Azotobacter $)$. At 80 DAS, there was significant difference between different treatments. Maximum plant height $(53.60 \mathrm{~cm})$ was obtained by the 
application of $\mathrm{T}_{10}(25 \%$ through $\mathrm{VC}+75 \%$ $\mathrm{N}$ through Urea + Azotobacter $)$, whereas the lowest value $246.33 \mathrm{~cm}$ in $\mathrm{T}_{3}(100 \% \mathrm{RDN}$ through VC). However, $\mathrm{T}_{8}(100 \% \mathrm{RDN}$ through $\mathrm{VC}+50 \% \mathrm{~N}$ through Urea + Azotobacter), and $\mathrm{T}_{2}(100 \% \mathrm{RDN}$ through Urea + Azotobacter) were found statistically at par with $\mathrm{T}_{10}(25 \%$ through $\mathrm{VC}+75 \% \mathrm{~N}$ through Urea + Azotobacter). Experimental finding showed that due to application of nitrogen at $120 \mathrm{~kg} \mathrm{ha}^{-1}$ over $80 \mathrm{~kg} \mathrm{ha}^{-1}$ attributed to significantly higher plant height, number of green leaves and tillers per plant in pearlmillet (Babu et al., 1995).

\section{Dry weight (gm)}

The observations of plant height are being presented in table 2 that reveals that there was a steady increase in the plant dry weight from 20 to 80 DAS. At 60 and 80 DAS were significant influence in plant height due to different treatments, while at 20 and 40 DAS non- significant. At $20 \mathrm{DAS}$, there was no significantly difference between different treatments. Maximum dry weight (2.03) was obtained by the application of $\mathrm{T}_{10}(100 \%$ RDN through Urea), whereas the lowest value $1.15 \mathrm{~cm}$ in $\mathrm{T}_{3}(100 \% \mathrm{RDN}$ through $\mathrm{VC})$. At $40 \mathrm{DAS}$, there was no significantly difference between different treatments. Maximum dry weight (8.39) was obtained by the application of $\mathrm{T}_{10}(25 \% \mathrm{RDN}$ through $\mathrm{VC}+75 \% \mathrm{~N}$ through Urea + Azotobacter), whereas the lowest value 6.38 in $\mathrm{T}_{3}(100 \% \mathrm{RDN}$ through VC). However, T9 (25\% RDN through VC + $75 \% \mathrm{~N}$ through Urea), $\mathrm{T}_{2}(100 \% \mathrm{RDN}$ through Urea), $\mathrm{T}_{3}(75 \% \mathrm{RDN}$ through $\mathrm{VC}+$ $25 \% \mathrm{~N}$ through Urea Azotobacter), were found statistically at par with $\mathrm{T}_{10}(25 \%$ through $\mathrm{VC}+75 \% \mathrm{~N}$ through Urea + Azotobacter). At 60 DAS, there was significant difference between different treatments. Maximum plant dry weight (59.13) was obtained by the application of $\mathrm{T}_{10}$ (25\% through $\mathrm{VC}+75 \% \mathrm{~N}$ through Urea + Azotobacter), whereas the lowest value 43.93 in $\mathrm{T}_{3}\left(100 \% \mathrm{RDN}\right.$ through VC). However, $\mathrm{T}_{9}$ (25\% RDN through $\mathrm{VC}+75 \% \mathrm{~N}$ through Urea), $\mathrm{T}_{2}$ (100\% RDN through Urea), $\mathrm{T}_{3}(75$ $\%$ RDN through $\mathrm{VC}+25 \% \mathrm{~N}$ through Urea Azotobacter), were found statistically at par with $\mathrm{T}_{10}(25 \%$ through $\mathrm{VC}+75 \% \mathrm{~N}$ through Urea + Azotobacter $)$. At 80 DAS, there was significant difference between different treatments. Maximum plant dry weight (105.30) was obtained by the application of $\mathrm{T}_{10}(25 \%$ through $\mathrm{VC}+75 \% \mathrm{~N}$ through Urea + Azotobacter), whereas the lowest value 98.83 in $\mathrm{T}_{3}(100 \% \mathrm{RDN}$ through $\mathrm{VC})$. However, $\mathrm{T}_{9}(25 \%$ RDN through $\mathrm{VC}+75 \%$ through Urea + Azotobacter $), \mathrm{T}_{2}(100 \% \mathrm{RDN}$ through Urea + Azotobacter), $\mathrm{T}_{4}(100 \% \mathrm{RDN}$ through $\mathrm{VC}+$ Azotobacter $), \mathrm{T}_{8}(50 \% \mathrm{RDN}$ through $\mathrm{VC}+50 \% \mathrm{~N}$ through Urea + Azotobacter), $\mathrm{T}_{5}(75 \% \mathrm{RDN}$ through $\mathrm{VC}+$ $25 \% \mathrm{~N}$ through Urea) were found statistically at par with $\mathrm{T}_{10}(25 \%$ through $\mathrm{VC}+75 \% \mathrm{~N}$ through Urea + Azotobacter). The probable reasons for recording higher stature of growth attributes viz., plant height, leaf area index, dry matter production and number of tillers m-2 was observed in different varieties due to increased levels of nitrogen with the application of $50 \mathrm{~kg} \mathrm{~N}$ ha-1. While all these parameters were at their lowest value with no nitrogen application in foxtail millet (Setaria italica L.) (AICRP Forage Crops, 2006).

\section{Tillers plant ${ }^{-1}$}

The observations of tillers plant ${ }^{-1}$ are being presented in table 3 . A perusal of the table 3 reveals that there was a steady increase in the tillers plant ${ }^{-1}$ from 20 to 80 DAS. At 20, 40, 60 and 80 DAS were significant influence on no. of tillers plant ${ }^{-1}$ due to different treatments. At 20 DAS, there was significantly difference between different treatments. Maximum tillers plant ${ }^{-1}$ (1.67) was obtained by the application of $\mathrm{T}_{10}(25 \%$ $\mathrm{RDN}$ through $\mathrm{VC}+75 \% \mathrm{~N}$ through Urea + Azotobacter), whereas the lowest value 0.73 in $\mathrm{T}_{1}(100 \% \mathrm{RDN}$ through Urea). However, 
$\mathrm{T}_{9}(25 \% \mathrm{RDN}$ through $\mathrm{VC}+75 \% \mathrm{~N}$ through Urea), was found statistically at par with $\mathrm{T}_{10}$ $(25 \%$ through $\mathrm{VC}+75 \% \mathrm{~N}$ through Urea + Azotobacter). At 40 DAS, there was significantly difference between different treatments. Maximum tillers plant ${ }^{-1}$ (2.40) was obtained by the application of $\mathrm{T}_{10}(25 \% \mathrm{RDN}$ through $\mathrm{VC}+75 \% \mathrm{~N}$ through Urea + Azotobacter), whereas the lowest value 1.27 in $\mathrm{T}_{3}\left(100 \% \mathrm{RDN}\right.$ through VC). However, $\mathrm{T}_{9}$ (25\% RDN through $\mathrm{VC}+75 \% \mathrm{~N}$ through Urea), $\mathrm{T}_{8}(50 \% \mathrm{RDN}$ through $\mathrm{VC}+50 \% \mathrm{~N}$ through Urea + Azotobacter), were found statistically at par with $\mathrm{T}_{10}(25 \%$ through VC $+75 \% \mathrm{~N}$ through Urea + Azotobacter). At 60 DAS, there was significant difference between different treatments. Maximum tillers plant $^{-1}$ (2.87) was obtained by the application of $\mathrm{T}_{10}(25 \%$ through $\mathrm{VC}+75 \%$ $\mathrm{N}$ through Urea + Azotobacter $)$, whereas the lowest value 1.60 in $\mathrm{T}_{1}(100 \% \mathrm{RDN}$ through Urea). However, $\mathrm{T}_{9}(25 \% \mathrm{RDN}$ through VC $+75 \% \mathrm{~N}$ through Urea), $\mathrm{T}_{8}(50 \% \mathrm{RDN}$ through $\mathrm{VC}+50 \% \mathrm{~N}$ through Urea + Azotobacter), were found statistically at par with $\mathrm{T}_{10}(25 \%$ through $\mathrm{VC}+75 \% \mathrm{~N}$ through Urea + Azotobacter $)$. At 80 DAS, there was significant difference between different treatments. Maximum tillers plant ${ }^{-1}$ (3.40) was obtained by the application of $\mathrm{T}_{10}(25 \%$ through $\mathrm{VC}+75 \% \mathrm{~N}$ through Urea + Azotobacter), whereas the lowest value 2.03 in $\mathrm{T}_{1}(100 \% \mathrm{RDN}$ through Urea). However, T9 $25 \%$ RDN through VC $+75 \%$ through Urea + Azotobacter), $\mathrm{T}_{8}(50 \% \mathrm{RDN}$ through $\mathrm{VC}+50 \% \mathrm{~N}$ through Urea + Azotobacter $)$, $\mathrm{T}_{6}(75 \% \mathrm{RDN}$ through $\mathrm{VC}+25 \% \mathrm{~N}$ through Urea + Azotobacter $), \mathrm{T}_{5}(75 \% \mathrm{RDN}$ through $\mathrm{VC}+25 \% \mathrm{~N}$ through Urea), $\mathrm{T}_{7}(50 \% \mathrm{RDN}$ through $\mathrm{VC}+50 \% \mathrm{~N}$ through Urea) were found statistically at par with $\mathrm{T}_{10}(25 \%$ through $\mathrm{VC}+75 \% \mathrm{~N}$ through Urea + Azotobacter). Experimental finding showed that the application of organic manures increased the number of effective tillers, grain weight per ear head and grain and biological yield of wheat over no organic manure. The probable reasons for recording higher stature of growth attributes viz., plant height, leaf area index, dry matter production and number of tillers m-2 was observed in different varieties due to increased levels of nitrogen with the application of $50 \mathrm{~kg} \mathrm{~N} \mathrm{ha}^{-1}$. While all these parameters were at their lowest value with no nitrogen application in foxtail millet (Setaria italica L.) (AICRP Forage Crops 2006).

\section{Crop Growth Rate (CGR) $\left(\mathrm{g} \mathrm{m}^{-1} \mathrm{day}^{-1}\right)$}

The observations of crop growth rate are being presented in table 4 . A perusal of table 4 reveals that there was a steady increase in the crop growth rate from 20 to 80 DAS. At 20, 40, 60 and 80 DAS were no significant influence in crop growth rate. At 0-20 DAS, there was no significantly difference between different treatments. Maximum crop growth rate $(0.10)$ was obtained by the application of $\mathrm{T}_{10}(100 \%$ RDN through $\mathrm{VC}+75 \% \mathrm{~N}$ through Urea+ Azotobacter), whereas the lowest value 0.05 in $\mathrm{T}_{3}(100 \% \mathrm{RDN}$ through VC). At 20-40 DAS, there was no significantly difference between different treatments. Maximum crop growth rate (8.39) was obtained by the application of $\mathrm{T}_{10}(25 \%$ $\mathrm{RDN}$ through $\mathrm{VC}+75 \% \mathrm{~N}$ through Urea + Azotobacter), whereas the lowest value 0.23 in $\mathrm{T}_{3}(100 \% \mathrm{RDN}$ through $\mathrm{VC})$. At $40-60$ DAS, there was no significant difference between different treatments. Maximum crop growth rate (2.53) was obtained by the application of $\mathrm{T}_{10}(25 \%$ through $\mathrm{VC}+75 \%$ $\mathrm{N}$ through Urea + Azotobacter), whereas the lowest value 1.82 in $\mathrm{T}_{3}$ (100\% RDN through $\mathrm{VC})$. At $80 \mathrm{DAS}$, there was no significant difference between different treatments. Maximum crop growth rate (2.74) was obtained by the application of $\mathrm{T}_{10}(25 \%$ through $\mathrm{VC}+75 \% \mathrm{~N}$ through Urea + Azotobacter), whereas the lowest value 2.29 in $\mathrm{T}_{1}(100 \%$ RDN through Urea). 
Table.1 Effect of organic and inorganic sources of nitrogen on plant height $(\mathrm{cm})$ in pearlmillet

\begin{tabular}{|c|c|c|c|c|c|}
\hline \multirow[b]{2}{*}{ No } & \multirow[b]{2}{*}{ Treatment } & \multicolumn{4}{|c|}{ Plant height(cm) } \\
\hline & & $\begin{array}{l}20 \\
\text { DAS }\end{array}$ & 40 DAS & $\begin{array}{c}60 \\
\text { DAS }\end{array}$ & $\begin{array}{c}80 \\
\text { DAS }\end{array}$ \\
\hline $\mathrm{T}_{1}$ & $100 \%$ RDN through Urea & 86.88 & 178.16 & 234.73 & 268.77 \\
\hline $\mathrm{T}_{2}$ & $100 \%$ RDN through Urea + Azotobacter & 86.22 & 181.17 & 230.10 & 270.19 \\
\hline $\mathrm{T}_{3}$ & $100 \%$ RDN through VC & 76.30 & 159.67 & 226.64 & 246.33 \\
\hline $\mathrm{T}_{4}$ & $100 \% \mathrm{RDN}$ through $\mathrm{VC}+$ Azotobacter & 81.93 & 168.30 & 229.27 & 261.57 \\
\hline $\mathrm{T}_{5}$ & $75 \% \mathrm{RDN}$ through $\mathrm{VC}+25 \% \mathrm{~N}$ through Urea & 83.83 & 176.80 & 220.67 & 252.97 \\
\hline $\mathrm{T}_{6}$ & $75 \% \mathrm{RDN}$ through $\mathrm{VC}+25 \% \mathrm{~N}$ through Urea + Azotobacter & 76.70 & 172.32 & 226.03 & 255.67 \\
\hline $\mathrm{T}_{7}$ & $50 \% \mathrm{RDN}$ through $\mathrm{VC}+50 \% \mathrm{~N}$ through Urea & 79.25 & 176.40 & 222.44 & 257.70 \\
\hline $\mathrm{T}_{8}$ & $50 \% \mathrm{RDN}$ through $\mathrm{VC}+50 \% \mathrm{~N}$ through Urea+ Azotobacter & 81.93 & 179.97 & 235.57 & 264.17 \\
\hline $\mathrm{T}_{9}$ & $25 \% \mathrm{RDN}$ through $\mathrm{VC}+75 \% \mathrm{~N}$ through Urea & 85.50 & 184.45 & 236.50 & 272.70 \\
\hline \multirow[t]{4}{*}{$\mathrm{T}_{10}$} & $25 \%$ RDN through $\mathrm{VC}+75 \% \mathrm{~N}$ through Urea+ Azotobacter & 84.67 & 189.34 & 243.33 & 277.17 \\
\hline & F- test & NS & S & S & $\mathrm{S}$ \\
\hline & S. Ed. $( \pm)$ & 7.66 & 5.74 & 5.42 & 3.45 \\
\hline & C. D. $(P=0.05)$ & - & 12.07 & 11.40 & 7.26 \\
\hline
\end{tabular}

Table.2 Effect of organic and inorganic sources of nitrogen on dry weight (gm) in pearlmillet

\begin{tabular}{|c|c|c|c|c|c|}
\hline \multirow[b]{2}{*}{ No } & \multirow[b]{2}{*}{ Treatment } & \multicolumn{4}{|c|}{ Dry weight (gm) } \\
\hline & & 20 & 40 & 60 & 80 \\
\hline $\mathrm{T}_{1}$ & $100 \%$ RDN through Urea & 1.55 & 6.95 & 46.60 & 98.87 \\
\hline $\mathrm{T}_{2}$ & $100 \%$ RDN through Urea + Azotobacter & 1.57 & 7.25 & 54.27 & 101.93 \\
\hline $\mathrm{T}_{3}$ & $100 \%$ RDN through VC & 1.15 & 6.38 & 43.93 & 98.83 \\
\hline $\mathrm{T}_{4}$ & $100 \% \mathrm{RDN}$ through $\mathrm{VC}+$ Azotobacter & 1.12 & 6.79 & 45.40 & 100.67 \\
\hline $\mathrm{T}_{5}$ & $75 \% \mathrm{RDN}$ through $\mathrm{VC}+25 \% \mathrm{~N}$ through Urea & 1.46 & 6.97 & 45.03 & 100.73 \\
\hline $\mathrm{T}_{6}$ & $75 \%$ RDN through $\mathrm{VC}+25 \% \mathrm{~N}$ through Urea + Azotobacter & 1.53 & 7.92 & 50.63 & 96.48 \\
\hline $\mathrm{T}_{7}$ & $50 \% \mathrm{RDN}$ through $\mathrm{VC}+50 \% \mathrm{~N}$ through Urea & 1.45 & 6.58 & 47.40 & 98.07 \\
\hline $\mathrm{T}_{8}$ & $50 \% \mathrm{RDN}$ through $\mathrm{VC}+50 \% \mathrm{~N}$ through Urea+ Azotobacter & 1.65 & 6.77 & 47.33 & 101.17 \\
\hline $\mathrm{T}_{9}$ & $25 \% \mathrm{RDN}$ through $\mathrm{VC}+75 \% \mathrm{~N}$ through Urea & 1.93 & 7.53 & 55.10 & 102.87 \\
\hline \multirow[t]{4}{*}{$\mathrm{T}_{10}$} & $25 \% \mathrm{RDN}$ through $\mathrm{VC}+75 \% \mathrm{~N}$ through Urea+ Azotobacter & 2.03 & 8.39 & 59.13 & 105.30 \\
\hline & F- test & NS & NS & $\mathrm{S}$ & $\mathrm{S}$ \\
\hline & S. Ed. $( \pm)$ & 0.43 & 0.56 & 3.80 & 2.24 \\
\hline & C. D. $(P=0.05)$ & - & - & 8.00 & 4.72 \\
\hline
\end{tabular}


Table.3 Effect of organic and inorganic sources of nitrogen on Tillers plant ${ }^{-1}$ of pearlmillet

\begin{tabular}{|c|c|c|c|c|c|}
\hline \multirow[b]{2}{*}{ No } & \multirow[b]{2}{*}{ Treatment } & \multicolumn{4}{|c|}{ Tillers plant $^{-1}$} \\
\hline & & $\begin{array}{l}20 \\
\text { DAS } \\
\end{array}$ & 40 DAS & $\begin{array}{c}\text { 60 } \\
\text { DAS } \\
\end{array}$ & $\begin{array}{c}\mathbf{8 0} \\
\text { DAS } \\
\end{array}$ \\
\hline $\mathrm{T}_{1}$ & $100 \%$ RDN through Urea & 0.73 & 1.53 & 1.60 & 2.03 \\
\hline $\mathrm{T}_{2}$ & $100 \%$ RDN through Urea + Azotobacter & 0.80 & 1.60 & 2.07 & 2.20 \\
\hline $\mathrm{T}_{3}$ & $100 \%$ RDN through VC & 0.93 & 1.27 & 1.87 & 2.27 \\
\hline $\mathrm{T}_{4}$ & $100 \%$ RDN through VC + Azotobacter & 0.87 & 1.40 & 1.73 & 2.60 \\
\hline $\mathrm{T}_{5}$ & $75 \% \mathrm{RDN}$ through $\mathrm{VC}+25 \% \mathrm{~N}$ through Urea & 0.80 & 1.40 & 1.93 & 2.87 \\
\hline $\mathrm{T}_{6}$ & $75 \%$ RDN through VC $+25 \% \mathrm{~N}$ through Urea + Azotobacter & 0.87 & 1.33 & 1.87 & 2.93 \\
\hline $\mathrm{T}_{7}$ & $50 \% \mathrm{RDN}$ through $\mathrm{VC}+50 \% \mathrm{~N}$ through Urea & 0.80 & 1.47 & 1.80 & 2.70 \\
\hline $\mathrm{T}_{8}$ & $50 \% \mathrm{RDN}$ through $\mathrm{VC}+50 \% \mathrm{~N}$ through Urea+ Azotobacter & 0.87 & 2.00 & 2.33 & 3.07 \\
\hline $\mathrm{T}_{9}$ & $25 \% \mathrm{RDN}$ through $\mathrm{VC}+75 \% \mathrm{~N}$ through Urea & 1.00 & 2.07 & 2.60 & 3.13 \\
\hline $\mathrm{T}_{10}$ & $25 \% \mathrm{RDN}$ through $\mathrm{VC}+75 \% \mathrm{~N}$ through Urea+ Azotobacter & 1.67 & 2.40 & 2.87 & 3.40 \\
\hline & F- test & $\mathrm{S}$ & $\mathrm{S}$ & S & $\mathrm{S}$ \\
\hline & S. Ed. (s \pm ) & 0.21 & 0.36 & 0.33 & 0.36 \\
\hline & C. D. $(P=0.05)$ & 0.46 & 0.77 & 0.70 & 0.76 \\
\hline
\end{tabular}

Table.4 Effect of organic and inorganic sources of nitrogen on Crop Growth Rate $\left(\mathrm{g} \mathrm{m}^{-2}\right.$ day $\left.^{-1}\right)$ of pearlmillet

\begin{tabular}{|c|c|c|c|c|c|}
\hline \multirow[b]{2}{*}{ No } & \multirow[b]{2}{*}{ Treatment } & \multicolumn{4}{|c|}{ Crop Growth Rate $\left(\mathrm{g} \mathrm{m}^{-2}\right.$ day $\left.^{-1}\right)$} \\
\hline & & $\begin{array}{l}\text { 0-20 } \\
\text { DAS } \\
\end{array}$ & $\begin{array}{l}\text { 0-20 } \\
\text { DAS }\end{array}$ & 0-20 DAS & 0-20 DAS \\
\hline $\mathrm{T}_{1}$ & $100 \%$ RDN through Urea & 0.08 & 0.26 & 1.82 & 2.29 \\
\hline $\mathrm{T}_{2}$ & $100 \%$ RDN through Urea + Azotobacter & 0.08 & 0.28 & 2.35 & 2.46 \\
\hline $\mathrm{T}_{3}$ & $100 \%$ RDN through VC & 0.06 & 0.23 & 1.82 & 2.31 \\
\hline $\mathrm{T}_{4}$ & $100 \% \mathrm{RDN}$ through $\mathrm{VC}+$ Azotobacter & 0.05 & 0.28 & 1.93 & 2.45 \\
\hline $\mathrm{T}_{5}$ & $75 \%$ RDN through VC $+25 \% \mathrm{~N}$ through Urea & 0.07 & 0.27 & 1.88 & 2.62 \\
\hline $\mathrm{T}_{6}$ & $75 \%$ RDN through $\mathrm{VC}+25 \% \mathrm{~N}$ through Urea + Azotobacter & 0.07 & 0.29 & 2.13 & 2.61 \\
\hline $\mathrm{T}_{7}$ & $50 \% \mathrm{RDN}$ through $\mathrm{VC}+50 \% \mathrm{~N}$ through Urea & 0.07 & 0.25 & 1.98 & 2.53 \\
\hline $\mathrm{T}_{8}$ & $50 \%$ RDN through VC $+50 \% \mathrm{~N}$ through Urea+ Azotobacter & 0.08 & 0.28 & 2.04 & 2.66 \\
\hline $\mathrm{T}_{9}$ & $25 \% \mathrm{RDN}$ through $\mathrm{VC}+75 \% \mathrm{~N}$ through Urea & 0.09 & 0.27 & 2.37 & 2.38 \\
\hline \multirow[t]{4}{*}{$\mathrm{T}_{10}$} & $25 \% \mathrm{RDN}$ through $\mathrm{VC}+75 \% \mathrm{~N}$ through Urea+ Azotobacter & 0.10 & 0.31 & 2.53 & 2.74 \\
\hline & F- test & NS & NS & NS & NS \\
\hline & S. Ed. $( \pm)$ & 0.02 & 0.03 & 0.23 & 0.24 \\
\hline & C. D. $(P=0.05)$ & - & - & - & - \\
\hline
\end{tabular}

Table.5 Effect of organic and inorganic sources of nitrogen on Relative Growth Rate $\left(\mathrm{g} \mathrm{g}^{-1}\right.$ day $\left.^{-1}\right)$ of pearlmillet

\begin{tabular}{|c|c|c|c|c|c|}
\hline \multirow{3}{*}{ No } & \multirow{3}{*}{ Treatment } & \multicolumn{4}{|c|}{ Relative Growth Rate $\left(\mathrm{g} \mathrm{g}^{-1}\right.$ day $\left.^{-1}\right)$} \\
\hline & & $\mathbf{0 - 2 0}$ & 0-20 & $\mathbf{0 - 2 0}$ & $\mathbf{0 - 2 0}$ \\
\hline & & DAS & DAS & DAS & DAS \\
\hline $\mathrm{T}_{1}$ & $100 \%$ RDN through Urea & 0.01 & 0.08 & 0.09 & 0.03 \\
\hline $\mathrm{T}_{2}$ & $100 \%$ RDN through Urea + Azotobacter & 0.02 & 0.08 & 0.10 & 0.03 \\
\hline $\mathrm{T}_{3}$ & $100 \%$ RDN through VC & 0.00 & 0.07 & 0.09 & 0.04 \\
\hline $\mathrm{T}_{4}$ & $100 \%$ RDN through $\mathrm{VC}+$ Azotobacter & 0.02 & 0.09 & 0.09 & 0.04 \\
\hline $\mathrm{T}_{5}$ & $75 \%$ RDN through VC $+25 \% \mathrm{~N}$ through Urea & 0.02 & 0.08 & 0.09 & 0.04 \\
\hline $\mathrm{T}_{6}$ & $75 \% \mathrm{RDN}$ through $\mathrm{VC}+25 \% \mathrm{~N}$ through Urea + Azotobacter & 0.02 & 0.07 & 0.09 & 0.03 \\
\hline $\mathrm{T}_{7}$ & $50 \%$ RDN through $\mathrm{VC}+50 \% \mathrm{~N}$ through Urea & 0.02 & 0.07 & 0.10 & 0.04 \\
\hline $\mathrm{T}_{8}$ & $50 \% \mathrm{RDN}$ through $\mathrm{VC}+50 \% \mathrm{~N}$ through Urea+ Azotobacter & 0.02 & 0.07 & 0.10 & 0.03 \\
\hline $\mathrm{T}_{9}$ & $25 \% \mathrm{RDN}$ through $\mathrm{VC}+75 \% \mathrm{~N}$ through Urea & 0.03 & 0.07 & 0.10 & 0.03 \\
\hline $\mathrm{T}_{10}$ & $25 \% \mathrm{RDN}$ through $\mathrm{VC}+75 \% \mathrm{~N}$ through Urea+ Azotobacter & 0.03 & 0.09 & 0.10 & 0.04 \\
\hline & F- test & NS & NS & NS & NS \\
\hline & S. Ed. $( \pm)$ & 0.060 & 0.016 & 0.005 & 0.004 \\
\hline & C. D. $(\mathrm{P}=0.05)$ & - & - & - & - \\
\hline
\end{tabular}


Table.6 Effect of organic and inorganic sources of nitrogen on ear plant ${ }^{-1}$ of pearlmillet

\begin{tabular}{|c|c|c|}
\hline & Treatment & Ear plant $^{-1}$ \\
\hline $\mathrm{T}_{1}$ & $100 \%$ RDN through Urea & 2.33 \\
\hline$T_{2}$ & $100 \%$ RDN through Urea + Azotobacter & 3.00 \\
\hline $\mathrm{T}_{3}$ & $100 \%$ RDN through VC & 2.67 \\
\hline $\mathrm{T}_{4}$ & $100 \%$ RDN through $\mathrm{VC}+$ Azotobacter & 3.00 \\
\hline $\mathrm{T}_{5}$ & $75 \% \mathrm{RDN}$ through $\mathrm{VC}+25 \% \mathrm{~N}$ through Urea & 2.39 \\
\hline $\mathrm{T}_{6}$ & $75 \%$ RDN through $\mathrm{VC}+25 \% \mathrm{~N}$ through Urea + Azotobacter & 2.73 \\
\hline $\mathrm{T}_{7}$ & $50 \%$ RDN through $\mathrm{VC}+50 \% \mathrm{~N}$ through Urea & 3.34 \\
\hline $\mathrm{T}_{8}$ & $50 \%$ RDN through $\mathrm{VC}+50 \% \mathrm{~N}$ through Urea+ Azotobacter & 3.13 \\
\hline $\mathrm{T}_{9}$ & $25 \%$ RDN through VC + $75 \% \mathrm{~N}$ through Urea & 3.34 \\
\hline$T_{10}$ & $25 \%$ RDN through $\mathrm{VC}+75 \% \mathrm{~N}$ through Urea+ Azotobacter & 3.57 \\
\hline & F-test & $\mathrm{S}$ \\
\hline & S. Ed. $( \pm)$ & 0.36 \\
\hline & C. D. $(P=0.05)$ & 0.76 \\
\hline
\end{tabular}

Table.7 Effect of organic and inorganic sources of nitrogen on length of ear $(\mathrm{cm})$ of pearlmillet

\begin{tabular}{|c|c|c|}
\hline & Treatment & Length of $\operatorname{Ear}(\mathrm{cm})$ \\
\hline $\mathrm{T}_{1}$ & $100 \%$ RDN through Urea & 26.07 \\
\hline $\mathrm{T}_{2}$ & $100 \%$ RDN through Urea + Azotobacter & 27.97 \\
\hline $\mathrm{T}_{3}$ & $100 \%$ RDN through VC & 26.73 \\
\hline $\mathrm{T}_{4}$ & $100 \%$ RDN through $\mathrm{VC}+$ Azotobacter & 27.53 \\
\hline $\mathrm{T}_{5}$ & $75 \% \mathrm{RDN}$ through $\mathrm{VC}+25 \% \mathrm{~N}$ through Urea & 26.53 \\
\hline $\mathrm{T}_{6}$ & $75 \% \mathrm{RDN}$ through $\mathrm{VC}+25 \% \mathrm{~N}$ through Urea + Azotobacter & 28.80 \\
\hline $\mathrm{T}_{7}$ & $50 \%$ RDN through VC + $50 \% \mathrm{~N}$ through Urea & 28.97 \\
\hline $\mathrm{T}_{8}$ & $50 \% \mathrm{RDN}$ through $\mathrm{VC}+50 \% \mathrm{~N}$ through Urea+ Azotobacter & 29.93 \\
\hline $\mathrm{T}_{9}$ & $25 \%$ RDN through $\mathrm{VC}+75 \% \mathrm{~N}$ through Urea & 30.63 \\
\hline $\mathrm{T}_{10}$ & $25 \%$ RDN through $\mathrm{VC}+75 \% \mathrm{~N}$ through Urea+ Azotobacter & 31.85 \\
\hline & F-test & $\mathrm{S}$ \\
\hline & S. Ed. $( \pm)$ & 1.26 \\
\hline & C. D. $(P=0.05)$ & 2.66 \\
\hline
\end{tabular}

Table.8 Effect of organic and inorganic sources of nitrogen on Length of no. of Grains ear ${ }^{-1}$ of pearlmillet

\begin{tabular}{clc}
\hline \multicolumn{1}{c}{ Treatment } & No. of Grains Ear $^{-1}$ \\
\hline $\mathrm{T}_{1}$ & $100 \%$ RDN through Urea & 2866.67 \\
$\mathrm{~T}_{2}$ & $100 \%$ RDN through Urea + Azotobacter & 3085.33 \\
$\mathrm{~T}_{3}$ & $100 \%$ RDN through VC & 2803.33 \\
$\mathrm{~T}_{4}$ & $100 \%$ RDN through VC + Azotobacter & 2760.00 \\
$\mathrm{~T}_{5}$ & $75 \%$ RDN through VC $+25 \% \mathrm{~N}$ through Urea & 2833.33 \\
$\mathrm{~T}_{6}$ & $75 \% \mathrm{RDN}$ through VC $+25 \% \mathrm{~N}$ through Urea + Azotobacter & 2966.67 \\
$\mathrm{~T}_{7}$ & $50 \% \mathrm{RDN}$ through VC $+50 \% \mathrm{~N}$ through Urea & 2715.67 \\
$\mathrm{~T}_{8}$ & $50 \% \mathrm{RDN}$ through VC $+50 \% \mathrm{~N}$ through Urea+ Azotobacter & 3099.00 \\
$\mathrm{~T}_{9}$ & $25 \% \mathrm{RDN}$ through VC $+75 \% \mathrm{~N}$ through Urea & 3233.33 \\
$\mathrm{~T}_{10}$ & $25 \% \mathrm{RDN}$ through VC $+75 \% \mathrm{~N}$ through Urea+ Azotobacter & 3433.33 \\
\hline & F-test & $\mathrm{S}$ \\
& S. Ed. $( \pm)$ & 206.96 \\
C. $\mathrm{D}(\mathrm{P}=0.05)$ & 434.84 \\
\hline
\end{tabular}


Table.9 Effect of organic and inorganic sources of nitrogen on Test weight (gm) of pearlmillet

\begin{tabular}{llc}
\hline \multicolumn{1}{c}{ Treatment } & Test weight (gm) \\
\hline $\mathrm{T}_{1}$ & $100 \%$ RDN through Urea & 7.15 \\
$\mathrm{~T}_{2}$ & $100 \%$ RDN through Urea + Azotobacter & 7.51 \\
$\mathrm{~T}_{3}$ & $100 \%$ RDN through VC & 7.86 \\
$\mathrm{~T}_{4}$ & $100 \%$ RDN through VC + Azotobacter & 7.94 \\
$\mathrm{~T}_{5}$ & $75 \%$ RDN through VC $+25 \% \mathrm{~N}$ through Urea & 7.75 \\
$\mathrm{~T}_{6}$ & $75 \%$ RDN through VC $+25 \% \mathrm{~N}$ through Urea + Azotobacter & 8.07 \\
$\mathrm{~T}_{7}$ & $50 \%$ RDN through VC $+50 \% \mathrm{~N}$ through Urea & 7.77 \\
$\mathrm{~T}_{8}$ & $50 \% \mathrm{RDN}$ through VC $+50 \% \mathrm{~N}$ through Urea+ Azotobacter & 8.31 \\
$\mathrm{~T}_{9}$ & $25 \% \mathrm{RDN}$ through VC $+75 \% \mathrm{~N}$ through Urea & 8.38 \\
$\mathrm{~T}_{10}$ & $25 \%$ RDN through VC $+75 \% \mathrm{~N}$ through Urea+ Azotobacter & 8.39 \\
\hline & F-test & $\mathrm{S}$ \\
S. Ed. $( \pm)$ & 0.35 \\
C. D. $(\mathrm{P}=0.05)$ & 0.74 \\
\hline
\end{tabular}

Table.10 Effect of organic and inorganic sources of nitrogen on Grain yield $\left(\mathrm{t} \mathrm{ha}^{-1}\right)$ of pearlmillet

\begin{tabular}{|c|c|c|}
\hline & Treatment & Grain yield $\left(\mathrm{t} \mathrm{ha}^{-1}\right)$ \\
\hline $\mathrm{T}_{1}$ & $100 \%$ RDN through Urea & 3.04 \\
\hline $\mathrm{T}_{2}$ & $100 \%$ RDN through Urea + Azotobacter & 3.29 \\
\hline $\mathrm{T}_{3}$ & $100 \%$ RDN through VC & 2.51 \\
\hline $\mathrm{T}_{4}$ & $100 \%$ RDN through VC + Azotobacter & 2.68 \\
\hline $\mathrm{T}_{5}$ & $75 \%$ RDN through $\mathrm{VC}+25 \% \mathrm{~N}$ through Urea & 3.11 \\
\hline $\mathrm{T}_{6}$ & $75 \%$ RDN through $\mathrm{VC}+25 \% \mathrm{~N}$ through Urea + Azotobacter & 3.14 \\
\hline $\mathrm{T}_{7}$ & $50 \%$ RDN through $\mathrm{VC}+50 \% \mathrm{~N}$ through Urea & 3.77 \\
\hline $\mathrm{T}_{8}$ & $50 \%$ RDN through VC $+50 \% \mathrm{~N}$ through Urea+ Azotobacter & 4.68 \\
\hline $\mathrm{T}_{9}$ & $25 \%$ RDN through $\mathrm{VC}+75 \% \mathrm{~N}$ through Urea & 4.72 \\
\hline $\mathrm{T}_{10}$ & $25 \%$ RDN through VC $+75 \% \mathrm{~N}$ through Urea+ Azotobacter & 4.97 \\
\hline & F-test & $\mathrm{S}$ \\
\hline & S. Ed. $( \pm)$ & 0.20 \\
\hline & C. D. $(P=0.05)$ & 0.43 \\
\hline
\end{tabular}

Table.11 Effect of organic and inorganic sources of nitrogen on Stover yield $\left(\mathrm{t} \mathrm{ha}^{-1}\right)$ of pearlmillet

\begin{tabular}{|c|c|c|}
\hline & Treatment & Stover yield $\left(\mathrm{t} \mathrm{ha}^{-1}\right)$ \\
\hline $\mathrm{T}_{1}$ & $100 \%$ RDN through Urea & 11.39 \\
\hline $\mathrm{T}_{2}$ & $100 \%$ RDN through Urea + Azotobacter & 11.51 \\
\hline $\mathrm{T}_{3}$ & $100 \%$ RDN through VC & 9.30 \\
\hline $\mathrm{T}_{4}$ & $100 \% \mathrm{RDN}$ through VC + Azotobacter & 9.33 \\
\hline $\mathrm{T}_{5}$ & $75 \% \mathrm{RDN}$ through $\mathrm{VC}+25 \% \mathrm{~N}$ through Urea & 10.15 \\
\hline $\mathrm{T}_{6}$ & $75 \% \mathrm{RDN}$ through $\mathrm{VC}+25 \% \mathrm{~N}$ through Urea + Azotobacter & 10.59 \\
\hline $\mathrm{T}_{7}$ & $50 \%$ RDN through $\mathrm{VC}+50 \% \mathrm{~N}$ through Urea & 11.01 \\
\hline $\mathrm{T}_{8}$ & $50 \%$ RDN through $\mathrm{VC}+50 \% \mathrm{~N}$ through Urea+ Azotobacter & 11.32 \\
\hline $\mathrm{T}_{9}$ & $25 \%$ RDN through $\mathrm{VC}+75 \% \mathrm{~N}$ through Urea & 12.67 \\
\hline$T_{10}$ & $25 \%$ RDN through $\mathrm{VC}+75 \% \mathrm{~N}$ through Urea+ Azotobacter & 12.99 \\
\hline & F-test & $\mathrm{S}$ \\
\hline & S. Ed. $( \pm)$ & 0.39 \\
\hline & C. D. $(P=0.05)$ & 0.82 \\
\hline
\end{tabular}


Table.12 Effect of organic and inorganic sources of nitrogen on harvest index (\%) of pearlmillet

\begin{tabular}{llc}
\hline \multicolumn{1}{c}{ Treatment } & Harvest index (\%) \\
\hline $\mathrm{T}_{1}$ & $100 \%$ RDN through Urea & 21.06 \\
$\mathrm{~T}_{2}$ & $100 \%$ RDN through Urea + Azotobacter & 22.22 \\
$\mathrm{~T}_{3}$ & $100 \%$ RDN through VC & 21.53 \\
$\mathrm{~T}_{4}$ & $100 \% \mathrm{RDN}$ through VC + Azotobacter & 22.31 \\
$\mathrm{~T}_{5}$ & $75 \% \mathrm{RDN}$ through VC $+25 \% \mathrm{~N}$ through Urea & 23.45 \\
$\mathrm{~T}_{6}$ & $75 \% \mathrm{RDN}$ through VC $+25 \% \mathrm{~N}$ through Urea + Azotobacter & 22.86 \\
$\mathrm{~T}_{7}$ & $50 \% \mathrm{RDN}$ through VC $+50 \% \mathrm{~N}$ through Urea & 25.50 \\
$\mathrm{~T}_{8}$ & $50 \% \mathrm{RDN}$ through VC $+50 \% \mathrm{~N}$ through Urea+ Azotobacter & 27.76 \\
$\mathrm{~T}_{9}$ & $25 \% \mathrm{RDN}$ through VC $+75 \% \mathrm{~N}$ through Urea & 27.14 \\
$\mathrm{~T}_{10}$ & $25 \%$ RDN through VC $+75 \% \mathrm{~N}$ through Urea+ Azotobacter & 29.25 \\
\hline & F-test & $\mathrm{S}$ \\
S. Ed. $( \pm)$ & 1.30 \\
C. $\mathrm{D} .(\mathrm{P}=0.05)$ & 2.74 \\
\hline
\end{tabular}

Table.13 Effect of organic and inorganic sources of nitrogen on Protein content $(\%)$ of pearlmillet

\begin{tabular}{llc}
\hline \multicolumn{1}{c}{ Treatment } & Protein content (\%) \\
\hline $\mathrm{T}_{1}$ & $100 \%$ RDN through Urea & 9.15 \\
$\mathrm{~T}_{2}$ & $100 \%$ RDN through Urea + Azotobacter & 9.64 \\
$\mathrm{~T}_{3}$ & $100 \%$ RDN through VC & 10.45 \\
$\mathrm{~T}_{4}$ & $100 \%$ RDN through VC + Azotobacter & 12.78 \\
$\mathrm{~T}_{5}$ & $75 \%$ RDN through VC $+25 \% \mathrm{~N}$ through Urea & 11.16 \\
$\mathrm{~T}_{6}$ & $75 \% \mathrm{RDN}$ through VC $+25 \% \mathrm{~N}$ through Urea + Azotobacter & 11.43 \\
$\mathrm{~T}_{7}$ & $50 \% \mathrm{RDN}$ through VC $+50 \% \mathrm{~N}$ through Urea & 10.75 \\
$\mathrm{~T}_{8}$ & $50 \% \mathrm{RDN}$ through VC $+50 \% \mathrm{~N}$ through Urea+ Azotobacter & 10.02 \\
$\mathrm{~T}_{9}$ & $25 \% \mathrm{RDN}$ through VC $+75 \% \mathrm{~N}$ through Urea & 11.42 \\
$\mathrm{~T}_{10}$ & $25 \% \mathrm{RDN}$ through VC $+75 \% \mathrm{~N}$ through Urea+ Azotobacter & 13.03 \\
\hline & F-test & $\mathrm{S}$ \\
& S. Ed. $( \pm)$ & 3.55 \\
C. $\mathrm{D} .(\mathrm{P}=0.05)$ & 2.45 \\
\hline
\end{tabular}

\section{Post-harvest observations of pearlmillet}

\section{Number of ear plant ${ }^{-1}$}

Tables 5 and 6 shows that there was significant effect of organic and inorganic sources of nitrogen on number of ear plant $^{-1}$. Maximum number of ear was found (3.57) with treatment $\mathrm{T}_{10}(25 \%$ through $\mathrm{VC}+75 \%$ $\mathrm{N}$ through Urea + Azotobacter $)$, while minimum number of ear (2.33) was observed in treatment $\mathrm{T}_{1}(100 \%$ RDN through $\mathrm{U})$, However $\mathrm{T}_{9}(25 \% \mathrm{RDN}$ through $\mathrm{VC}+75$ $\% \mathrm{~N}$ through Urea), $\mathrm{T}_{8}(50 \% \mathrm{RDN}$ through $\mathrm{VC}+50 \% \mathrm{~N}$ through Urea + Azotobacter $), \mathrm{T}_{4}$ (100\%RDN through Urea + Azotobacter $), \mathrm{T}_{2}$ (100 \% RDN through Urea)were found statistically at par with $\mathrm{T}_{10}(25 \%$ through $\mathrm{VC}$
$+75 \% \mathrm{~N}$ through Urea + Azotobacter $)$. Experimental findings showed that due the application of organic manure might be attributed to the better nutrient availability and its favorable effect on soil physical and biological properties resulting increased growth of plant and grain and straw yield (Rathor et al., 2008).

\section{Length of ear (cm)}

Table 7 shows that there was significant effect of organic and inorganic sources of nitrogen on length of ear $(\mathrm{cm})$. Maximum length of ear was found (31.85) with treatment $\mathrm{T}_{10}(25 \%$ through $\mathrm{VC}+75 \% \mathrm{~N}$ through Urea + Azotobacter), while minimum length of ear(26.07) was observed in treatment $\mathrm{T}_{1}(100$ 
$\%$ RDN through Urea),However $\mathrm{T}_{9}(25 \%$ RDN through $\mathrm{VC}+75 \% \mathrm{~N}$ through Urea), $\mathrm{T}_{8}(50 \% \mathrm{RDN}$ through $\mathrm{VC}+50 \% \mathrm{~N}$ through Urea + Azotobacter), were found statistically at par with $\mathrm{T}_{10}(25 \%$ through $\mathrm{VC}+75 \% \mathrm{~N}$ through Urea + Azotobacter). Experimental finding showed that application of nitrogen @ $120 \mathrm{~kg} / \mathrm{ha}$ was at par with $160 \mathrm{~kg} \mathrm{~N} / \mathrm{ha}$, significantly increased crude protein content over control, 40 and $80 \mathrm{~kg} \mathrm{~N} / \mathrm{ha}$. in pearlmillet (Rathor et al., 2008).

\section{Number of grains ear ${ }^{-1}$}

Table 8 shows that there was significant effect of organic and inorganic sources of nitrogen on number of grains ear ${ }^{-1}$. Maximum number of grains ear ${ }^{-1}$ was found (3433.33)with treatment $\mathrm{T}_{10}(25 \%$ through $\mathrm{VC}+75 \% \mathrm{~N}$ through Urea + Azotobacter), while minimum number of grains ear ${ }^{-1}(2803.33)$ was observed in treatment $\mathrm{T}_{3}(100 \% \mathrm{RDN}$ through VC),However $\mathrm{T}_{9}(25 \%$ RDN through $\mathrm{VC}$ $+75 \% \mathrm{~N}$ through Urea), $\mathrm{T}_{8}(50 \% \mathrm{RDN}$ through $\mathrm{VC}+50 \% \quad \mathrm{~N}$ through Urea + Azotobacter), were found statistically at par with $\mathrm{T}_{10}(25 \%$ through $\mathrm{VC}+75 \% \mathrm{~N}$ through Urea + Azotobacter). Experimental finding showed that increase in yield attributes might be due to the effective potential of pearmillet is decided by growth and yield component. This was reflected in the present study (Rathor et al., 2008).

\section{Test weight (gm)}

Table 9 shows that there was significant effect of organic and inorganic sources of nitrogen on test weight. Maximum test weight was found (8.39) with treatment $\mathrm{T}_{10}(25 \%$ through $\mathrm{VC}+75 \% \mathrm{~N}$ through Urea + Azotobacter $)$, while lowest (7.15) was observed in treatment $\mathrm{T}_{1}\left(100 \%\right.$ RDN through Urea). However $\mathrm{T}_{9}$ (25\% RDN through $\mathrm{VC}+75 \% \mathrm{~N}$ through Urea), $\mathrm{T}_{8}(50 \% \mathrm{RDN}$ through $\mathrm{VC}+50 \% \mathrm{~N}$ through Urea + Azotobacter $), \mathrm{T}_{6}(75 \% \mathrm{RDN}$ through $\mathrm{VC}+25 \% \mathrm{~N}$ through Urea + Azotobacter were found statistically at par with $\mathrm{T}_{10}(25 \%$ through $\mathrm{VC}+75 \% \mathrm{~N}$ through Urea + Azotobacter). Experimental finding showed that a dose of $90 \mathrm{~kg} \mathrm{~N}^{-1}$ applied in 2 equal splits resulted in taller plants and more tillers plant $^{-1}$ and 1000 grains weight which in turn in higher grain yield and stover yield (Kumawat and Jat, 2007).

\section{Grain yield ( $\mathrm{t} \mathrm{ha}^{-1}$ )}

Table 10 shows that there was significant effect of organic and inorganic sources of nitrogen on grain yield. Maximum grain yield was found (4.97) with treatment $\mathrm{T}_{10}(25 \%$ through $\mathrm{VC}+75 \% \mathrm{~N}$ through Urea + Azotobacter), while minimum grain yield(2.51) was observed in treatment $\mathrm{T}_{3}(100$ $\%$ RDN through Urea),However $\mathrm{T}_{9}(25 \%$ RDN through $\mathrm{VC}+75 \% \mathrm{~N}$ through Urea), $\mathrm{T}_{8}$ (50 \% RDN through $\mathrm{VC}+50 \% \mathrm{~N}$ through Urea + Azotobacter), were found statistically at par with $\mathrm{T}_{10}(25 \%$ through $\mathrm{VC}+75 \% \mathrm{~N}$ through Urea + Azotobacter). Experimental findings showed that due the application of organic manure might be attributed to the better nutrient avaibility and its favorable effect on soil physical and biological properties resulting increased yield attributes and straw yield further, vermicompost increase the efficiency of added chemical fertilizers in soil and increased rate of humification.Humic acid in vermicompost enhances the availability of both native and added micronutrients in soil and thus plant yield attributes and yield increased (Singh et al., 2005, Yadav and Solanki 2002).

\section{Stover yield (t ha $\left.{ }^{-1}\right)$}

Table 11 shows that there was significant effect of organic and inorganic sources of nitrogen on stover yield $\left(\mathrm{t} \mathrm{ha}{ }^{-1}\right)$. Maximum stover yield $\left(\mathrm{t} \mathrm{ha}^{-1}\right)$ was found (12.99) with treatment $\mathrm{T}_{10}(25 \%$ through $\mathrm{VC}+75 \% \mathrm{~N}$ 
through Urea + Azotobacter), while minimum stover yield (9.30) was observed in treatment $\mathrm{T}_{3}$ (100\% RDN through VC). However $\mathrm{T}_{9}$ (25\% RDN through $\mathrm{VC}+75 \% \mathrm{~N}$ through Urea) was found statistically at par with $\mathrm{T}_{10}(25 \%$ through $\mathrm{VC}+75 \% \mathrm{~N}$ through Urea + Azotobacter). Experimental findings showed that due the application of organic manure might be attributed to the better nutrient availability and its favorable effect on soil physical and biological properties resulting increased growth of plant and grain and straw yield (Rathor et al., 2008).

\section{Harvest index (\%)}

Table 12 shows that there was significant effect of organic and inorganic sources of nitrogen on harvest index $(\%)$. Maximum harvest index (\%) was found (27.67)with treatment $\mathrm{T}_{10}(25 \%$ through $\mathrm{VC}+75 \% \mathrm{~N}$ through Urea + Azotobacter), while minimum harvest index (21.06) was observed in treatment $\mathrm{T}_{1}(100 \%$ RDN through Urea), However $\mathrm{T}_{8}(50 \%$ RDN through $\mathrm{VC}+50 \%$ $\mathrm{N}$ through Urea + Azotobacter $), \mathrm{T}_{9}(25 \%$ RDN through $\mathrm{VC}+75 \% \mathrm{~N}$ through Urea), $\mathrm{T}_{7}$ (50 \% RDN through $\mathrm{VC}+50 \%$ through Urea), were found statistically at par with $\mathrm{T}_{10}$ (25\% through $\mathrm{VC}+75 \% \mathrm{~N}$ through Urea + Azotobacter). Experimental findings showed that due the application of organic manure might be attributed to the better nutrient availability and its favorable effect on soil physical and biological properties resulting increased growth of plant, grain yield and straw yield (Rathore et al., 2008).

\section{Protein content $(\%)$}

Table 13 shows that there was significant effect of organic and inorganic sources of nitrogen on protein content (\%). Maximum protein content (\%) was found (13.04) with treatment $\mathrm{T}_{10}(25 \%$ through $\mathrm{VC}+75 \% \mathrm{~N}$ through Urea + Azotobacter), while minimum protein content (9.15) was observed in treatment $\mathrm{T}_{1}(100 \%$ RDN through Urea), However $\mathrm{T}_{4}(100 \%$ RDN through $\mathrm{VC}+$ Azotobacter), $\mathrm{T}_{6}(75 \% \mathrm{RDN}$ through $\mathrm{VC}+$ $25 \%$ through Urea + Azotobacter $), \mathrm{T}_{9}(25 \%$ RDN through $\mathrm{VC}+75 \% \mathrm{~N}$ through Urea), $\mathrm{T}_{5}$ (75\% RDN through VC $+25 \%$ through Urea + Azotobacter $), \mathrm{T}_{7}(50 \% \mathrm{RDN}$ through $\mathrm{VC}+$ $50 \%$ through Urea), were found statistically at par with $\mathrm{T}_{10}(25 \%$ through $\mathrm{VC}+75 \% \mathrm{~N}$ through Urea + Azotobacter). Experimental finding showed that protein content was highest when 75 per cent of RDF was combined with vermicompost at $10 \mathrm{t} \mathrm{ha}^{-1}$ in pearlmillet-hybrid napier (Jayanthi et al., (2002 a).

In conclusion, effect of organic and inorganic sources of nitrogen under in treatment $\mathrm{T}_{10}$ (25\% RDN through $\mathrm{VC}+75 \% \mathrm{~N}$ through Urea + Azotobacter) recorded maximum plant height $(277.17 \mathrm{~cm})$, plant dry weight (105.30g), tillers plant ${ }^{-1}(3.40)$, crop growth rate $\left(2.74 \mathrm{~g} \mathrm{~m}^{-2}\right.$ day $\left.^{-1}\right)$, relative growth rate (0.04 $\mathrm{g} \mathrm{g}^{-1}$ day $\left.^{-1}\right)$, ear plant ${ }^{-1}$ (3.57), length of ear (31.85), no. of grains ear ${ }^{-1}(3433.33)$, test weight $(8.39 \mathrm{~g})$, grain yield $\left(4.97 \mathrm{t} \mathrm{ha}^{-1}\right)$, straw yield $\left(12.99 \mathrm{t} \mathrm{ha}^{-1}\right)$, protein content $(13.03 \%)$ and harvest index (27.67\%). Whereas the lowest value in terms of plant height $(246.33 \mathrm{~cm})$, dry weight $(98.83 \mathrm{~g})$, number of grains (2803.33 $\left.\mathrm{ear}^{-1}\right)$ grain yield $\left(2.51 \mathrm{t} \mathrm{ha}^{-1}\right)$ and stover yield $\left(9.30 \mathrm{t} \mathrm{ha}^{-1}\right)$ was observed in the treatment $\mathrm{T}_{3}$ i.e., (100\% RDN through VC). Further, number of tillers (2.03 plant $\left.^{-1}\right)$, crop growth rate $\left(2.29 \mathrm{~g} \mathrm{~m}^{-2}\right.$ day $\left.^{-1}\right)$, relative growth rate $\left(0.03 \mathrm{~g} \mathrm{~g}^{-1} \mathrm{day}^{-1}\right)$, number of ear (2.33 plant $\left.^{-1}\right)$, length of ear $(26.07 \mathrm{~cm})$, test weight $(7.15 \mathrm{~g})$, protein content $(9.15 \%)$, harvest index $(21.06 \%)$ was recorded in the treatment $\mathrm{T}_{1}(100 \% \mathrm{RDN}$ through Urea).

\section{Acknowledgements}

The authors are thankful to the Head, Department of Agronomy, Sam Higginbottom Institute of Agriculture, Technology and Sciences (Formerly Allahabad Agricultural 
Institute), (Deemed to-be-University), Allahabad (U.P), India for providing facilities to carry out the present study.

\section{References}

AICRP-Forage Crops, 2006. Effect of nitrogen levels on promising entries of pearlmillet. All India Co-ordinated Research Project on forage crops. IGFRI Jhansi, Annual Report Kharif2005: 161177.

Anonymous 2010-11. Annual Report All India Co-ordinated Pearlmillet Improvement Project. pp. 141-142.

Babu, R.S., Patil, G. T. S. and Prabhakar, A.S. 1995. Effect of stage of cutting, nitrogen and phosphorus level on forage pearlmillet (Pennisetum glaucum L.). Forage Research, 20: 225 - 231.

Behera, U. K., Pradhan, S. and Sharma, A.R. 2007. Effect of Integrated Nutrient Management practices on productivity of durum wheat (Triticum durum L.) in the vertisols of central India. Indian Journal of Agriculture Science, 77 (10): 635-8.

Brown, R.F. and Mayer, D.G. 1988. Representing cumulative germination. 1. A critical analysis of single-value germination indices. Annals of Botany, 61: 117-125.

Donald, C.M. 1962. In search of yield. Journal of Australian. Institute of Agriculture Science, 28: 171-178.

Jayanthi, C., Malarvizhi, P., Chinnusamy, C. and Mythili, S. 2002a. Integrated nutrient management for bajra-hybrid napier system. Journal of Farming System Research and Development, 8(12): 10-14.

Kumawat, P.D. and Jat, N.L. 2007. Effect of organic manure and nitrogen fertilization on productivity of barley. Indian Journal of Agronomy, 50 (3): 200-202.

Rathore, V.S., Singh, P. and Gautam, R.C. 2008. Productivity and water use efficiency of rainfed pearlmillet (Pennisetum glaucum L.) as influenced by planting patterns and integrated nutrient management. Indian Journal of Agronomy, 51(1): 46-48.

Singh, J. and Singh, K.P. 2005. Effect of organic manures and herbicides on yield and yield attributing characters of wheat. Indian Journal of Agronomy, 50 (4): 289-291.

Singh, R.C., Kumar, S., Kadian, V.S. and Singh, S. N. 2005. Effect of FYM and fertilizer along and their combination on yield of pearlmillet. Haryana Agricultural University. Journal of Research, 35: 109-112

Sonawane, P.D., Wadile, S.C., Girase, P.P., Chitodkar, S.S. and Sonawane, D.A. 2010. Response of summer pearlmillet (Pennisetum glauchum L.) to depth and time of irrigation. Scheduling. International Journal of Agricultural Sciences, 6(1): 283-285.

Yadava, N.S. and Solanki, N.S. 2002. Effect of levels of nitrogen and its time of application on fodder production of pearl millet. Forage Research, 28: 6-7

\section{How to cite this article:}

Shobha Kumari, Vikram Singh, Neha and Preeti Choudhary. 2017. Study the Effect of Organic and Inorganic Sources of Nitrogen on Growth and Yield of Kharif Pearl Millet. Int.J.Curr.Microbiol.App.Sci. 6(8): 769-782. doi: https://doi.org/10.20546/ijcmas.2017.608.098 